\title{
Detection of Aldolase Activity on Polyacrylamide Gels: Application to 2-Keto-4-hydroxyglutarate
} Aldolase $^{1}$

\author{
Neil D. Lewinski and Eugene E. DekKeR² \\ Department of Biological Chemistry, The University of Michigan, \\ Ann Arbor, Michigan 48109
}

Received August 15, 1977; accepted January 3, 1978

\begin{abstract}
A method is described for the detection of 2-keto-4-hydroxyglutarate aldolase activity after electrophoresis of the enzyme on polyacrylamide gels. When gels are incubated with substrate (2-keto-4-hydroxyglutarate), activity is seen as a yellow-colored band due to interaction of the product (glyoxylate) with ortho-aminobenzaldehyde and glycine. Positive results have been obtained using either crude cell-free preparations or homogeneous enzyme from Escherichia coli as well as with highly purified samples of aldolase from bovine liver or kidney extracts. The method is potentially applicable to other aldolases that liberate an aliphatic aldehyde as a product; modifications and limitations of the procedure for detecting fructose 1,6-diphosphate aldolase, 2-keto-3-deoxy-6phosphogluconate aldolase, and 2-deoxyribose-5-phosphate aldolase activities have been explored.
\end{abstract}

The aldol-type reaction is an important and common method, both chemically and biochemically, for lengthening or shortening the carbon chains of organic molecules. 2-Keto-4-hydroxyglutarate aldolase (2-oxo4-hydroxyglutarate glyoxylate-lyase) catalyzes the aldolytic cleavage or formation of 2-keto-4-hydroxyglutarate (KHG): ${ }^{3}$

$$
\mathrm{KHG} \rightleftharpoons \text { pyruvate }+ \text { glyoxylate }
$$

This reaction is an important terminal step in the catabolism of L-hydroxyproline by mammals $(1-3)$. KHG-aldolase has been highly purified from extracts of rat liver (4,5), bovine liver (6), and Escherichia coli (7); the enzyme obtained from any of these sources is a Class I (Schiff-base mechanism) aldolase. Studies so far completed with this enzyme have demonstrated that it has numerous atypical properties when compared

${ }^{1}$ This work was supported by a research grant (AM-03718) from the National Institute of Arthritis, Metabolism, and Digestive Diseases, U. S. Public Health Service.

${ }^{2}$ Author to whom correspondence should be addressed.

${ }^{3}$ Abbreviations used in this work: KHG, 2-keto-4-hydroxyglutarate; KDPG, 2-keto-3deoxy-6-phosphogluconate; FDP, fructose 1,6-diphosphate. 
with other aldolases, and an examination of its molecular and catalytic properties has provided new insights into enzymatic aldolytic catalysis.

Over the past several years, a method has been sought for detecting KHG-aldolase activity on polyacrylamide gels, particularly since there has been the indication that there might be different molecular forms of the enzyme (6). The report of Soda et al. (8) on the spectrophotometric determination of glyoxylate by reaction with ortho-aminobenzaldehyde and glycine has been adapted to visualize this aldolase activity following electrophoresis. The applicability of this staining procedure to other aldolases has also been explored.

\section{MATERIALS AND METHODS}

Chemicals. DL-2-Keto-4-hydroxyglutaric acid was prepared by nonenzymatic transamination of threo-4-hydroxy-DL-glutamate (3); threohydroxy-DL-glutamic acid was synthesized as described previously (9). Solutions of ortho-aminobenzaldehyde (Sigma Chemical Co.) were prepared fresh as needed. Solutions of sodium glyoxylate and sodium pyruvate (Sigma Chemical $\mathrm{Co}$.) as well as fructose 1,6 -diphosphate $\cdot 3 \mathrm{Na}^{+} \cdot 7 \mathrm{H}_{2} \mathrm{O}$ (Calbiochem) were stored at $-15^{\circ} \mathrm{C}$. 2-Deoxyribose-5-phosphate $\cdot \mathrm{Ba}^{2+}$ (Calbiochem) was prepared for use as follows: $19.4 \mathrm{mg}$ were dissolved in 1.5 $\mathrm{ml}$ of $\mathrm{H}_{2} \mathrm{O}$; dry Dowex $50-\mathrm{X} 8$ resin $\left(2.6 \mathrm{~g}, 200-400 \mathrm{mesh}, \mathrm{H}^{4}\right.$ phase) was added, and the mixture was swirled for $1 \mathrm{~min}$; after the resin was quickly removed by filtration, the resultant acidic solution was titrated to $\mathrm{pH} 6.0$ with $\mathrm{KOH}$ solution and stored frozen. DL-Glyceraldehyde 3-phosphate diethylacetal $\cdot \mathrm{Ba}^{2+}$ and dihydroxyacetone phosphate dimethyl ketal (Calbiochem) were prepared for use according to directions provided by the supplier for hydrolysis of short-chain sugar phosphates.

Acrylamide, $N, N^{\prime}$-methylene-bis-acrylamide (Bis), and $N, N, N^{\prime}, N^{\prime}$ tetramethylethylenediamine (TEMED) were electrophoresis grade (Bio-Rad Laboratories); Tris and glycine were from Sigma Chemical Co., reagent grade persulfate and aniline blue black (Acid Black I) were from Matheson Coleman and Bell; Bacto agar was from Difco Laboratories; and Sepraphore III cellulose polyacetate strips were from Gelman Instrument Co.

Potassium glycinate, $\mathrm{pH} 8.2$, was made according to the method of Yorifuji et al. (10). Tris-glycine clectrophoresis buffer, $\mathrm{pH} 8.5$, contained $2.25 \mathrm{~g}$ of Tris and $10.8 \mathrm{~g}$ of glycine per $750 \mathrm{ml}$. Glass double-distilled $\mathrm{H}_{2} \mathrm{O}$ was used for the preparation of all reagents and solutions.

Proteins. 2-Keto-4-hydroxyglutarate aldolase (KHG-aldolase) was purified from extracts of $E$. coli K-12 (11) and bovine liver (12). The enzyme was also partially purified from extracts of bovine kidney. ${ }^{4}$

${ }^{4}$ R. P. Kitson and E. E. Dekker, unpublished work. 
Rabbit muscle fructose 1,6-diphosphate aldolase (FDP-aldolase) (Sigma Chemical $\mathrm{Co}$.) was diluted with or dialyzed against $50 \mathrm{~mm}$ glycine$\mathrm{KCl}, \mathrm{pH} 8.2$, before use. 2-Deoxyribose-5-phosphate aldolase of Salmonella typhimurium was a generous gift from Dr. P. A. Hoffee, University of Pittsburgh. 2-Keto-3-deoxy-6-phosphogluconate aldolase (KDPG-aldolase) from Pseudomonas putida and the substrate (KDPG) were graciously provided by Dr. H. P. Meloche, Papanicolaou Cancer Research Institute, Miami, and Dr. W. A. Wood, Michigan State University, East Lansing. The protein content of enzyme solutions was determined by the procedure of Lowry et al. (13) with serum albumin as a standard.

Electrophoresis. The procedure of Davis (14) for the preparation of polyacrylamide gels was followed, utilizing $7 \%$ separating gels at $\mathrm{pH} 8.8$ to 9.0. Tubes of 5-mm internal diameter and gels $7 \mathrm{~cm}$ in length were used. Protein samples were usually applied to a portion of the stacking gel and allowed to polymerize before electrophoresis. On occasion, samples were layered on the stacking gel in $10 \%$ sucrose $(\mathrm{w} / \mathrm{v})$ under the cathode buffer. Electrophoresis was carried out at 3 to $5^{\circ} \mathrm{C}$ in a Canalco Model 6 electrophoresis chamber with Tris-glycine, $\mathrm{pH} 8.5$, and a constant current of 4 $\mathrm{mA} /$ tubc. When tracking dye $(0.005 \%$ solution of bromophenol blue) placed in the upper cathode chamber had nearly traversed the length of the gel, the gels were removed and the position of the dye was marked with a short length of wire. Gels were stored in $7 \%$ acetic acid.

For cellulose acetate electrophoresis, the directions provided by Gelman Instrument $\mathrm{Co}$. were followed. Sepraphore III cellulose polyacetate strips $(1 \times 6.75$ in.) were presoaked in Gelman high resolution electrophoresis buffer, blotted between two strips of moist filter paper, and placed in the electrophoresis chamber already equilibrated with the same buffer. Samples containing 10 to $70 \mu \mathrm{g}$ of protein were applied with a Gelman serum applicator to a line of origin lightly marked with pencil. Electrophoresis was performed for $1 \mathrm{hr}$ at $360 \mathrm{~V}$ and $2 \mathrm{~A}$.

Protein staining procedures. Polyacrylamide gels were stained with a $0.5 \%$ solution of aniline blue black in $7 \%$ acetic acid for $1 \mathrm{hr}$, followed by destaining for $30 \mathrm{~min}$ with a Canalco Model 20 electrophoretic destainer.

A solution of $0.5 \mathrm{~g}$ of aniline blue black and $5.0 \mathrm{~g}$ of $\mathrm{HgCl}_{2}$ in $5 \%$ acetic acid was used to stain the cellulose polyacetate strips. Subsequently, the strips were rinsed three times in fresh solutions containing glacial acetic acid $(2 \mathrm{ml})$, glycerol $(15 \mathrm{ml})$, and distilled $\mathrm{H}_{2} \mathrm{O}(100 \mathrm{ml})$. After the strips were rinsed in $5 \%$ acetic acid $(100 \mathrm{ml})$ followed by distilled $\mathrm{H}_{2} \mathrm{O}(100 \mathrm{ml})$, they were cleared with $10 \%$ acetic acid in anhydrous methanol and finally mounted on microscope slides.

Adolase activity staining techniques. The method of Soda et al. (8) for the spectrophotometric determination of glyoxylic acid was modified for detecting KHG-aldolase activity on polyacrylamide gels. After electrophoresis, the gels were washed with distilled $\mathrm{H}_{2} \mathrm{O}$ several times and then 
placed in $10 \times 75-\mathrm{mm}$ test tubes containing $1.0 \mathrm{ml}$ of $1 \mathrm{M}$ glycine $-\mathrm{KCl}, \mathrm{pH}$ $8.2,0.5 \mathrm{ml}$ of $25 \mathrm{~mm}$ ortho-aminobenzaldehyde, and $0.4 \mathrm{ml}$ of $50 \mathrm{~mm}$ DLKHG, pH 6.5. After the gels were incubated for 30 to $90 \mathrm{~min}$ at $37^{\circ} \mathrm{C}$, they were removed from the reaction mixture, rinsed with distilled $\mathrm{H}_{2} \mathrm{O}$ and scanned at $440 \mathrm{~nm}$ in distilled $\mathrm{H}_{2} \mathrm{O}$.

For the detection of enzymatic activity following cellulose polyacetate electrophoresis, a procedure was developed in which agar slides containing the necessary assay components were placed in contact with the strips containing the electrophoresed samples. The agar slides were prepared as follows: Bacto agar $(1 \mathrm{~g})$ was dissolved in $50 \mathrm{ml}$ of boiling $1 \mathrm{M}$ glycine$\mathrm{KCl}, \mathrm{pH} 8.2$. After the mixture was cooled to approximately $65^{\circ} \mathrm{C}, 25 \mathrm{mg}$ of ortho-aminobenzaldehyde and $25 \mathrm{mg}$ of FDP were added and dissolved. Approximately $2-\mathrm{ml}$ portions of this still hot solution were quickly applied to clean microscope slides. When cooled, these agar-coated slides were wrapped in foil to exclude light and stored at 2 to $4^{\circ} \mathrm{C}$ in a closed, humidified environment. When used, cellulose polyacetate strips were sandwiched between an agar-coated and a plain glass slide. After this setup stands for about $1 \mathrm{hr}$ at room temperature, the colored band associated with enzymatic activity can be photographed and/or compared directly to strips stained for protein.

Spectrophotometric measurement of enzymatic activities and scanning of gels. The activity of the KDPG-aldolase provided to us was checked by the procedure of Hammerstedt et al. (15). When the method of Soda et al. (8) was applied to measuring KDPG-aldolase activity in solution, the assay mixture contained the following components: $0.5 \mathrm{ml}$ of $1 \mathrm{M}$ glycine- $\mathrm{KCl}, \mathrm{pH} 8.2 ; 0.25 \mathrm{ml}$ of $25 \mathrm{~mm}$ ortho-aminobenzaldehyde; 0.05 $\mathrm{ml}$ of $36 \mathrm{~mm}$ KDPG, and $0.19 \mathrm{ml}$ of water. These components were temperature equilibrated to $37^{\circ} \mathrm{C}$, and the reaction was initiated by adding approximately $10 \mu \mathrm{g}$ of KDPG-aldolase (the final reaction volume was 1.0 $\mathrm{ml}$ ). The progress of the reaction was followed at $440 \mathrm{~nm}$ with a thermostatted Gilford Model 2000 recording spectrophotometer equipped with an automatic cuvette positioner. When other aldolase activities were measured by the method of Soda et al. (8), the following amounts of substrate and enzyme were used: for FDP-aldolase, $0.05 \mathrm{ml}$ of $0.1 \mathrm{M} \mathrm{FDP}$ and 20-100 $\mu \mathrm{g}$ of protein in a final reaction volume of $1.8 \mathrm{ml}$; for KHG-aldolase, 0.40 $\mathrm{ml}$ of $50 \mathrm{~mm}$ DL-KHG and approximately $10 \mu \mathrm{g}$ of purified enzyme in a final volume of $2.0 \mathrm{ml}$. In these assays, no activity was observed in the absence of either the substrate or ortho-aminobenzaldehyde, and the color response diminished considerably when glycine was replaced by alanine, substantiating the requirement for an $\omega$-amino acid (8).

Scans of protein-stained and activity-stained gels were performed at 560 and $440 \mathrm{~nm}$, respectively, using the same spectrophotometer equipped with a Model 2410-S linear transport and a Gilford Model 2412 quartz gel cuvette with a $1.0-\mathrm{cm}$ path length. 


\section{RESULTS AND DISCUSSION}

The spectrophotometric method of Soda et al. (8) for the determination of glyoxylate is based on the appearance of a yellow color, presumably due to the dehydration of ortho-aminobenzaldehyde, an $\omega$-amino acid (i.e., glycine), and glyoxylate, with formation of a dihydroquinazolinium compound $(16,17)$. In earlier work, Albrecht, Scher, and Vogel (18) demonstrated that formaldehyde, propionaldehyde, butyraldehyde, and $n$ valeraldehyde gave similar responses when reacted with ortho-aminobenzaldehyde and methylamine. Insignificant or slight responses were observed with chloral hydrate, glucose, acetone, $\alpha$-ketoglutarate, pyruvate, or benzaldehyde. These results suggested to us that the quantitative procedure of Soda et al. (8) might not only be applicable to KHG-aldolase, which catalyzes glyoxylate formation [Reaction (1)], but also to other aldolases whose aldolytic products include an appropriate aliphatic aldehyde (i.e., glyceraldehyde-3-phosphate which, without exception, is one of the products formed by FDP-, KDPG- and 2-deoxyribose-5-phosphate aldolases). Figure 1 shows that in this procedure glyceraldehyde-3phosphate, like glyoxylate, does indeed give a linear response with increasing concentrations; so also does 2-deoxyribose-5-phosphate. With each of these three compounds, the presumed yellow-colored dihydroquinazolinium compounds exhibit absorption maxima at approximately $440 \mathrm{~nm}$. When applied to other substrates or products utilized or formed by aldolases of interest in this study, the following compounds were found to give no sig-

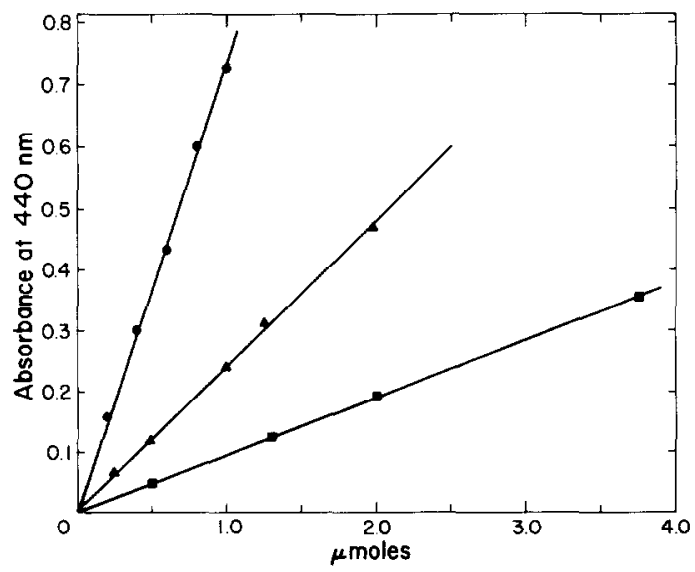

FIG. 1. Relationship (in micromoles) between $(\bullet)$ glyoxylate, $(\Delta)$ glyceraldehyde-3phosphate, ( 2-deoxyribose-5-phosphate and color intensity at $440 \mathrm{~nm}$. The reaction mixtures uniformly contained $1.0 \mathrm{ml}$ of $1 \mathrm{M}$ glycine- $\mathrm{KCl}, \mathrm{pH} 7.8,1.0 \mathrm{ml}$ of $25 \mathrm{mM}$ ortho-aminobenzaldehyde, and varying amounts of the aldehyde solution to be tested. Final volume was made to $3 \mathrm{ml}$ with $\mathrm{H}_{2} \mathrm{O}$. Reaction mixtures were heated at $37^{\circ} \mathrm{C}$ for $15 \mathrm{~min}$; absorbance measurements were made with a Gilford Model 2000 spectrophotometer, 1-cm light path. 
nificant color response when tested at relevant concentrations: KHG, pyruvate, FDP, dihydroxyacetone, and KDPG. As a consequence, and as can be seen in Fig. 2, the spectrophotometric method of Soda et al. (8) can be readily adapted to measure the rates of the reaction catalyzed by either KHG-aldolase, KDPG-aldolase, or FDP-aldolase.

Figure 3 shows the patterns obtained on polyacrylamide gels when crude cell-free preparations of $E$. coli are stained for the presence of proteins or KHG-aldolase activity and also illustrates that the zone of catalytically-active material in essentially homogeneous preparations is coincident with the overwhelmingly predominant protein-stainable band. Results identical to those shown in Fig. 3 were also obtained with highly purified preparations of KHG-aldolase from bovine liver and kidney. Most of the conditions designed to uptimize this stain on gels for KHG-aldolase activity were established using the homogeneous enzyme from $E$. coli.

Under standard reaction conditions, the intensity of the activity stain on gels reaches a maximum after approximately $90 \mathrm{~min}$ of incubation. The yellow band corresponding to KHG-aldolase activity is stable for over

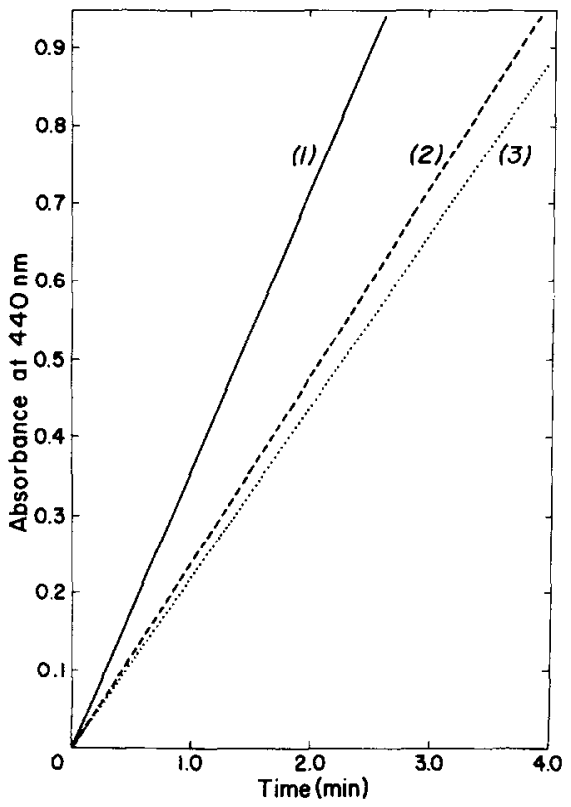

Fig. 2. Rates of reactions catalyzed by (1) KHG-aldolase, (2) FDP-aldolase and (3) KDPGaldolase. Reaction conditions were as follows: (1) $2.0 \mathrm{ml}$ final volume containing $1000 \mu \mathrm{mol}$ of glycine-KCl, pH 8.4, $12.5 \mu \mathrm{mol}$ of ortho-aminobenzaldehyde, $20 \mu \mathrm{mol}$ of DL-KHG, $\mathrm{pH} 6.5$, and $10 \mu \mathrm{g}$ of purified enzyme; incubation, $37^{\circ} \mathrm{C} ;(2) 1.8 \mathrm{ml}$ final volume containing $100 \mu \mathrm{g}$ of FDP-aldolase, $5 \mu \mathrm{mol}$ of FDP, $12.5 \mu \mathrm{mol}$ of ortho-aminobenzaldehyde, and $1000 \mu \mathrm{mol}$ of glycine- $\mathrm{KCl}, \mathrm{pH} 8.2$; incubation, $25^{\circ} \mathrm{C}$; (3) $1.0 \mathrm{ml}$ final volume containing $500 \mu \mathrm{mol}$ of glycine $-\mathrm{KCl}$, pH 8.2, $6.25 \mu \mathrm{mol}$ of ortho-aminobenzaldehyde, $2 \mu \mathrm{mol}$ of $\mathrm{KDPG}$, and $10 \mu \mathrm{g}$ of aldolase; incubation, $37^{\circ} \mathrm{C}$. Addition of enzyme initiated the reaction in each case. 

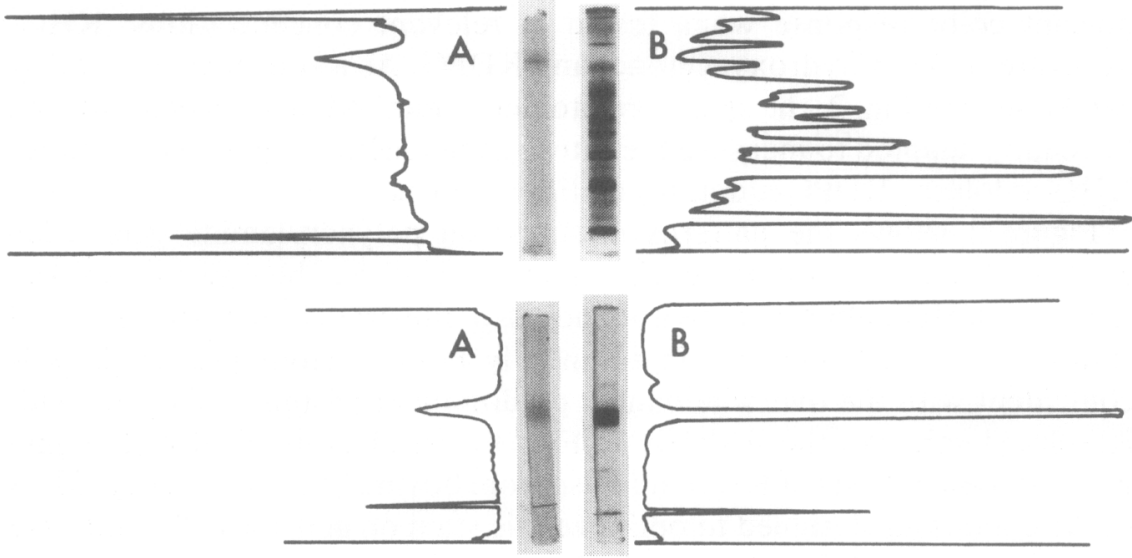

FIG. 3. Electrophoretic pattern of a crude preparation ( $300 \mu \mathrm{g}$, upper gels and scans) and of essentially homogeneous $E$. coli KHG-aldolase (150 $\mu \mathrm{g}$, lower gels and scans) on polyacrylamide: (A) stained for enzymatic activity, scanned at $440 \mathrm{~nm}$; (B) stained for protein with aniline blue black; scanned at $560 \mathrm{~nm}$. Migration was from top (cathode) to bottom (anode); thin wire segments at the bottom of the gels designate the position of tracking dye. For photographic purposes, a blue Corning glass filter (No. 4-72) was routinely used to accentuate the yellow activity band.

$3 \mathrm{hr}$ when stored in the reaction mixture at $37^{\circ} \mathrm{C}$. If the gel is placed in distilled water, the yellow-colored zone completely diffuses within 15 to 20 $\mathrm{min}$. We have found that the gels may be scanned (approx. $7 \mathrm{~min}$ per gel) and then returned to the reaction mixture at $37^{\circ} \mathrm{C}$ with only a minimal decrease in staining intensity in subsequent scans.

The intensity of the activity stain, as measured by the peak height of individual scans, varies with substrate concentration, as shown in Fig. 4. The

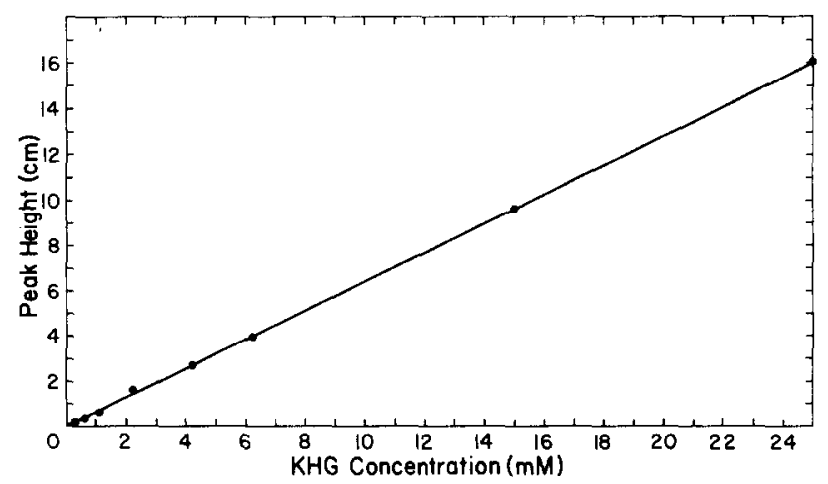

FIG. 4. Effect of KHG concentration on E. coli KHG-aldolase activity following polyacrylamide gel electrophoresis; $75 \mu \mathrm{g}$ of enzyme were used. Maximum peak heights of gel scans at $440 \mathrm{~nm}$ are plotted; a peak height of $1 \mathrm{~cm}$ corresponds to an absorbance of 0.044 . 
level of KHG used in the standard reaction mixture ( $10.5 \mathrm{~mm}$ final concentration) was an arbitrary compromise between color intensity and the supply of the compound available to us. An ortho-aminobenzaldehyde concentration of $6.6 \mathrm{~mm}$ approaches the lower limit of maximal effectiveness but was chosen so as to minimize background color due to its diffusion into the gels.

Since KHG-aldolase from either $E$. coli or bovine liver has a $\mathrm{pH}$ optimum of 8.6 to 8.8 , the $\mathrm{pH}$ of the glycine- $\mathrm{KCl}$ buffer was raised from 7.8 [as used by Soda et al. (8)] to 8.2 in an effort to maximize color intensity and enzyme activity. The electrophoretic procedure was also performed at 2 to $5^{\circ} \mathrm{C}$ in order to decrease the possibility of heat denaturation of the aldolase. The minimal level of KHG-aldolase which can be detected on gels under standard reaction conditions is in the range of 2 to $4 \mu \mathrm{g}$ of protein $(0.1-0.2$ units). Quite probably, this level of sensitivity can be increased at the expense of higher concentrations of the substrate.

\section{Applicability to Some Other Aldolases}

Attempts were made to apply this staining technique to 2-deoxyribose5-phosphate aldolase and KDPG-aldolase, since an aldolytic cleavage product of both enzymes (i.e., glyceraldehyde-3-phosphate) responds in the method of Soda et al. (8) (see Fig. 1). In the former case, however, 2 deoxyribose-5-phosphate also reacts significantly with ortho-aminobenzaldehyde and glycine; hence the technique is not applicable in this instance. Although KDPG-aldolase activity could be readily followed in solution by the spectrophotometric method of Soda et al. (8) (Fig. 2), limited attempts to demonstrate this aldolase activity on polyacrylamide gels were not successful. Extensive efforts to develop appropriate conditions were not made due to limitations of both KDPG-aldolase and its substrate. We also attempted to apply the method to fructose 1,6-diphosphate aldolase since, as indicated earlier, glyceraldehyde-3-phosphate responds to the colorimetric reaction of Soda et al. (8) but FDP and dihydroxyacetone phosphate do not. Several factors, however, contributed to abortive attempts to demonstrate this particular aldolase activity on polyacrylamide gels following electrophoresis. FDP-aldolase exhibits unusually low electrophoretic mobility, entering the gels only a maximum of about $5 \mathrm{~mm}$ even after long periods of electrophoresis. Furthermore, the resultant band of protein is poorly defined, and attempts to detect the FDP-aldolase activity of these electrophoresed samples were uniformly unsuccessful. Neither prior electrophoresis of the gels to remove persulfate nor utilization of lower concentrations of polyacrylamide in the gels altered these experimental findings; attempts to recover activity from appropriate segments of gels macerated in buffer were also unsuccessful. Since we found that macerated samples of ordinary polyacrylamide gel inhibit FDP-aldolase activity, it 


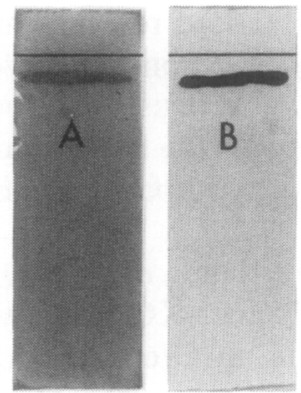

FIG. 5. Electrophoretic pattern of FDP-aldolase $(100 \mu \mathrm{g})$ on cellulose acetate strips. Enzyme was applied at the penciled line; migration was from top (cathode) to bottom (anode). The strips were stained for (A) activity and (B) protein as described in the Materials and Methods section.

seems possible that under the conditions employed polyacrylamide gels cause aggregation and/or inactivation of this enzyme.

As an alternative, we investigated the electrophoresis of FDP-aldolase on cellulose polyacetate strips. When the procedure described in Materials and Methods was followed, FDP-aldolase activity was initially observed by incubating the cellulose acetate strips directly in the required assay mixture. A better visualization method was sought, however, since the yellow reaction product diffused quickly into the surrounding fluid. Use of agar slides containing the assay components proved effective in localizing the zone of FDP-aldolase activity, and the position of the protein band correlates well with this activity stain as shown in Fig. 5 .

The method described, therefore, is a reliable and highly sensitive method for detecting KHG-aldolase activity on polyacrylamide gels and can be adapted to visualize FDP-aldolase activity after electrophoresis on strips of cellulose polyacetate.

\section{REFERENCES}

1. Adams, E., and Goldstone, A. (1960) J. Biol. Chem. 235, 3492-3498.

2. Adams, E., and Goldstone, A. (1960) J. Biol. Chem. 235, 3504-3512.

3. Maitra, U., and Dekker, E. E. (1963) J. Biol. Chem. 238, 3660-3669.

4. Maitra, U., and Dekker, E. E. (1964) J. Biol. Chem. 239, 1485-1491.

5. Adams, E. (1971) in Methods in Enzymology (Colowick, S. P., and Kaplan, N. O., eds.), Vol. 17B, pp. 280-284, Academic Press, New York.

6. Kobes, R. D., and Dekker, E. E. (1969) J. Biol. Chem. 244, 1919-1925.

7. Nishihara, H., and Dekker, E. E. (1972) J. Biol. Chem. 247, 5079-5087.

8. Soda, K., Toyama, S., Misono, H., Hirasawa, T., and Asada, K. (1970) Agr. Biol. Chem. 37, $1393-1400$.

9. Dekker, E. E., and Maitra, U. (1962) J. Biol. Chem. 237, 2218-2227.

10. Yorifuji, T., Ogata, K., and Soda, K. (1971) J. Biol. Chem. 246, 5085-5092.

11. Dekker, E. E., Nishihara, H., and Grady, S. R. (1975) in Methods in Enzymology (Colowick, S. P., and Kaplan, N. O., eds.), Vol. 42, pp. 285-290, Academic Press, New York. 
12. Dekker, E. E., Kobes, R. D., and Grady, S. K. (1975) in Methods in Enzymology (Colowick, S. P., and Kaplan, N. O., eds.), Vol. 42, pp. 280-285, Academic Press, New York.

13. Lowry, O. H., Rosebrough, N. J., Farr, A. L., and Randall, R. J. (1951) J. Biol. Chem. 193, 265-275.

14. Davis, B. J. (1964) Ann. N. Y. Acad. Sci. 121, 404-442.

15. Hammerstedt, R. H., Möhler, H., Decker, K. A., Ersfeld, D., and Wood, W. A. (1975) in Methods in Enzymology (Colowick, S. P., and Kaplan, N. O., eds.), Vol. 42, pp. 258-264, Academic Press, New York.

16. Schopf, C., Komzak, A., Braun, F., and Jacobi, E. (1948) Ann. 559, 1-42.

17. Vogel, H. J., and Davis, B. D. (1952) J. Amer. Chem. Soc. 74, 109-112.

18. Albrecht, A. M.. Scher, W. I., and Vogel, H. J. (1962) Anal. Chem. 34, 398-400. 